\title{
5.2 RADIO POLARIZATION OF THE CRAB NEBULA
}

\author{
R. G. CONWAY \\ University of Manchester, Nuffield Radio Astronomy Laboratories, Jodrell Bank, U.K.
}

\begin{abstract}
Optical and radio measurements of polarization can be combined to find the configuration of the magnetic field throughout the Crab Nebula. The component in the line of sight is found from the radio rotation measure.

New measurements of polarisation at $11 \mathrm{~cm}$ with a resolution of $7^{\prime \prime} \times 14^{\prime \prime}$ are combined with previous results to show that the rotation measure is fairly uniform and near $-25 \mathrm{rad} \mathrm{m}^{-2}$ near the centre, but that it becomes irregular near the edges. It rises to $300 \mathrm{rad} \mathrm{m}^{-2}$ in the filaments, possibly indicating a concentration of electrons about $10-100 \mathrm{~cm}^{-3}$.
\end{abstract}

The Crab Nebula is of interest historically on two counts: not only was it the first radio source to be identified with an optical object, it was also the first source in which the synchrotron mechanism was identified, by virtue of the linear polarization of the optical radiation. Figure 1 shows the map at $5^{\prime \prime}$ arc resolution obtained by Woltjer, giving the fractional polarization $m$ and the position angle $\chi$, quantities which are related to the Stokes parameters $I, Q$ and $U$ by the expression for the 'polarized intensity'.

$$
m I \exp (2 j \chi)=Q+j U .
$$

The central portion of the nebula exhibits a more or less aligned polarization, with the $E$ vectors running roughly parallel to the major axis of the nebula. The magnetic field thus appears to be predominantly along the minor axis direction. Surrounding the central area are a series of 'fans' in each of which the $E$ vectors are arranged radially about a centre. Here the percentage polarization is high, and may exceed $50 \%$. For the most part the fans are related to prominent filaments, which are seen by Woltjer as the seat of powerful currents, the magnetic field of which is arranged radially around the filament throughout a considerable volume. The polarization is not related to filaments however at the north end of the nebula.

No radio measurements yet exist at as high a resolution as the optical map. In principle the radio observations could complement the optical, since the optical polarization determines the direction of $B_{\perp}$, while the radio maps which are subject to modification by Faraday rotation, could give information on $B_{\|}$. In practice, however, the information on $B_{\|}$is still incomplete and statistical rather than detailed. We shall discuss the information gained by studies of progressively finer resolution.

Burn (1966) has considered the integrated polarization of the nebula as a whole. The value of $m$ decreases rapidly from $9 \%$ (optical) to a few percent (at $\lambda 3 \mathrm{~cm}$ ) thereafter decreasing slowly with wavelength. Burn assumes that this variation is caused by the Faraday effect, and is due to the fact that some parts of the nebula suffer more 


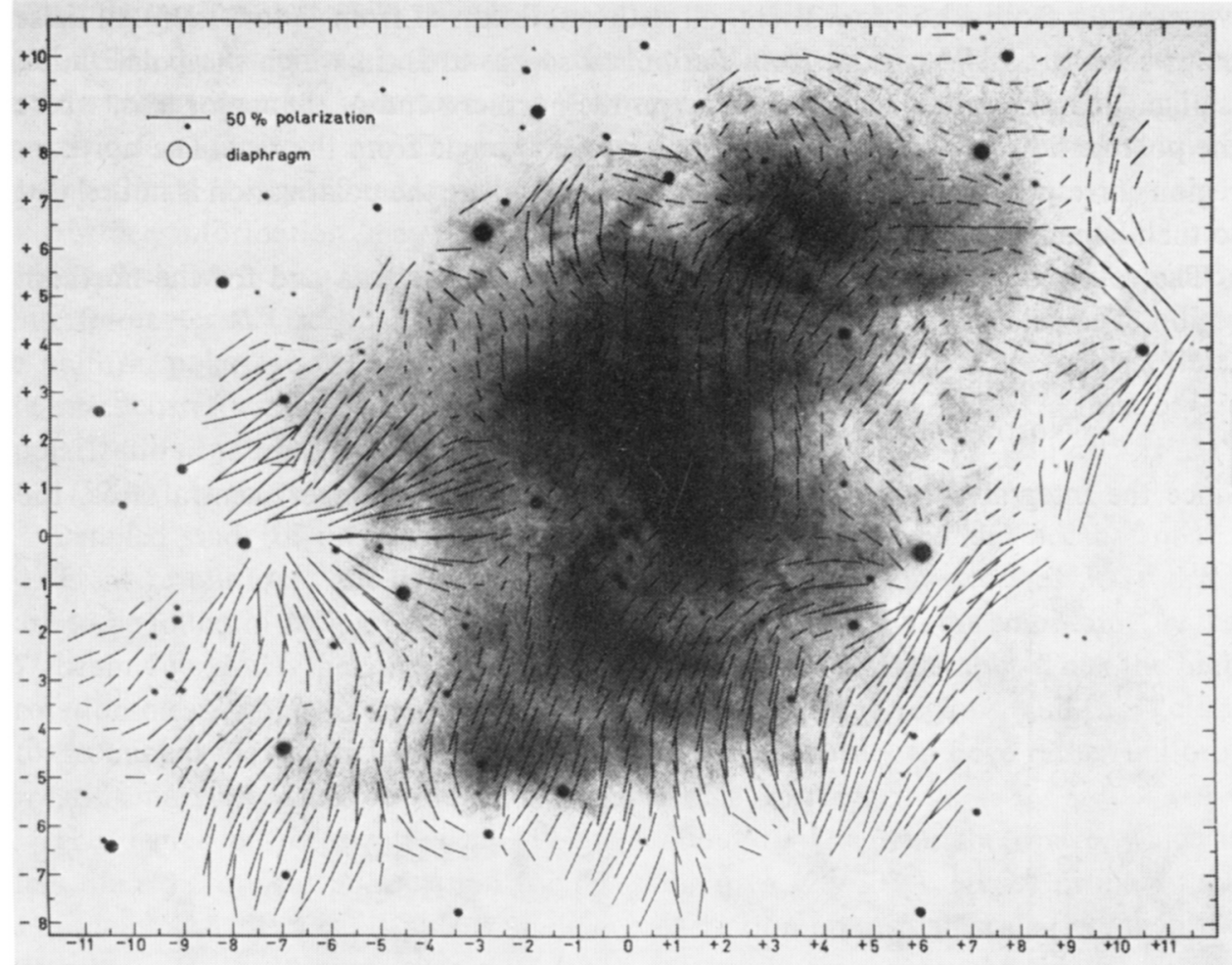

Fig. 1. Optical polarization of the Crab Nebula. The origin is at RA $05^{\mathrm{h}} 31^{\mathrm{m}} 31.5^{\mathrm{s}}, \operatorname{Dec} 21^{\circ} 58^{\prime} 55^{\prime \prime}$, and the unit of distance is $11^{\prime \prime}$ arc.

rotation than others. If the usual definition of rotation measure is adopted (Gardner and Whiteoak, 1966):

$$
R M=8 \times 10^{5} \int N_{e} B \mathrm{~d} l \text { radians } / m^{2}
$$

then we may define a 'Faraday dispersion', $\Delta$, as the range of rotation measures present from greatest to least. Burn shows that the variation of $m$ with $\lambda$ is determined principally by the dispersion, $\Delta$, and deduces that the value of $\Delta$ for the Crab is $\sim 1000 \mathrm{rad} / \mathrm{m}^{2}$. Order of magnitude estimates for $N_{e}$ and $B$ in or around the filaments allow values of this order, though with considerable uncertainty.

Burn's assumption that the $m(\lambda)$ curve is entirely due to Faraday rotation may be questioned, since it is known that the electrons producing the radio radiation are distributed over a wider volume than those producing the optical radiation. Basically, as the radio data become available, one wishes to repeat Burn's analysis, point by point, across the face of the nebula, to overcome this objection.

Low-resolution maps of the nebula have been published by Downs and Thompson (1968), by Mayer and Hollinger (1967), by Allen (1967) and by Wright (1970) at 
wavelengths from $\lambda 1.55$ to $\lambda 21 \mathrm{~cm}$, all with resolution of from $1^{\prime}$ to $2^{\prime}$ arc. All these maps show a central region, from the pulsar southwards, in which the polarization is aligned, and another region, centred on the northern end of the major axis, where the polarization has a markedly different position angle from the rest. The northern region corresponds to the area on the optical map where the polarization is not related to the filaments.

The rotation measure may be deduced for the central area and for the northern region. The values are

$$
\begin{aligned}
& \text { Centre }-25 \mathrm{rad} / \mathrm{m}^{2} \\
& \text { North }-40 \mathrm{rad} / \mathrm{m}^{2} .
\end{aligned}
$$

Since the integrated polarization is determined by the (stronger) central area, the

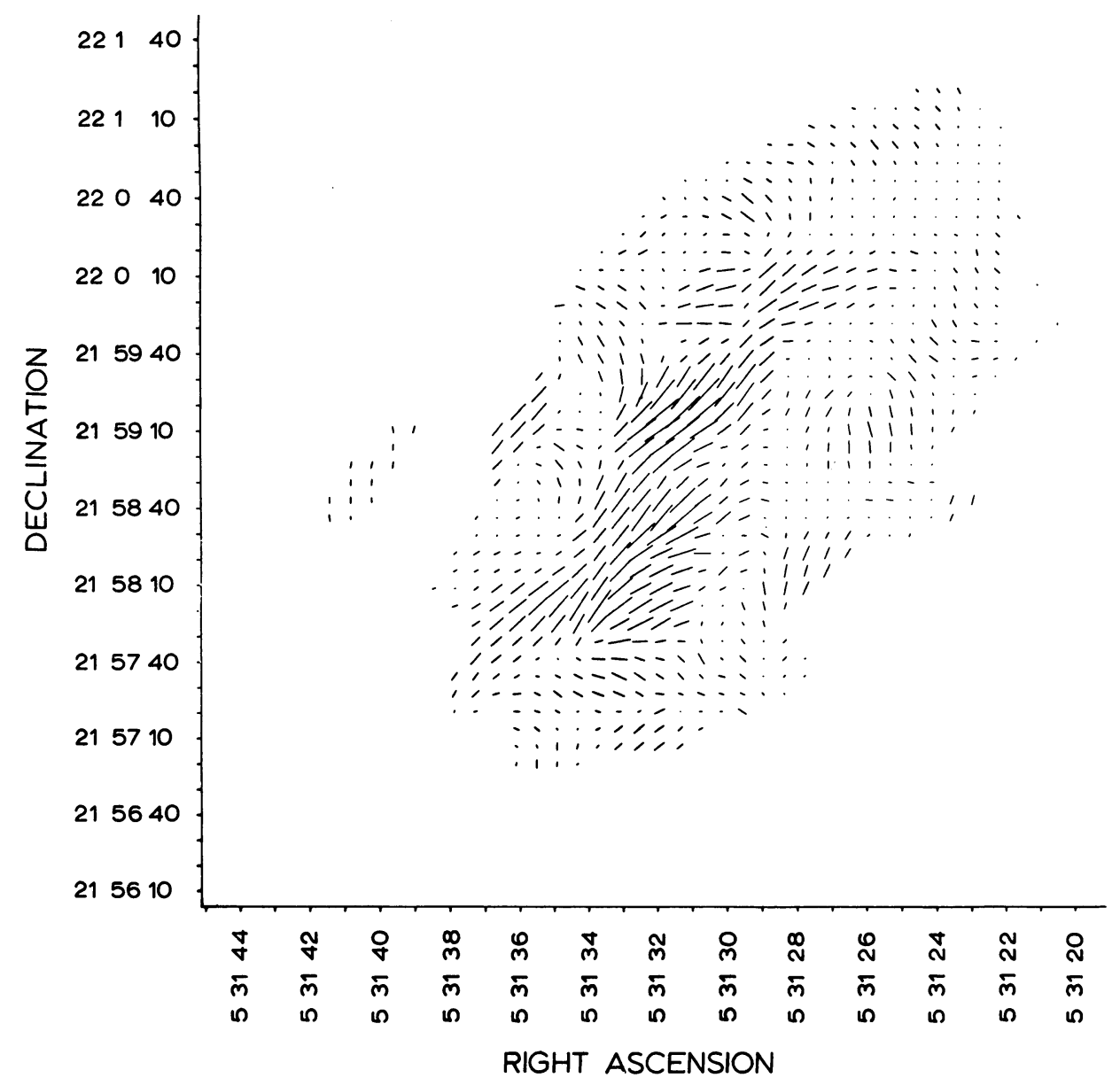

Fig. 2. Radio polarization of the Crab Nebula at $\lambda 11 \mathrm{~cm}$. The bar lines are proportional in length to the polarized intensity, $m I$. The beam is $8^{\prime \prime} \times 14^{\prime \prime}$, with the larger dimension roughly parallel to the major axis of the nebula. 
value of $R M$ for the centre is closely equal to that deduced by Gardner and Davies (1966) for the integrated polarization. Since this value is compatible with values for other nearby sources, Gardner and Davies ascribe the value $-25 \mathrm{rad} / \mathrm{m}^{2}$ to the galactic Faraday rotation. The net rotation due to the nebula is unknown, but we may estimate it as $<50 \mathrm{rad} / \mathrm{m}^{2}$.

Further information may be gained from a high resolution study at $\lambda 11 \mathrm{~cm}$. Figure 2 shows a map, with resolution $8^{\prime \prime} \times 14^{\prime \prime}$, obtained with the NRAO 3-element interferometer at Greenbank, U.S.A., in 1967. As before, the central area has rather a uniform polarization. The northern region can also be seen, and for the first time, features corresponding to the great optical fans can be made out. These are areas of low polarization, notably two areas centred at $\alpha 05^{\mathrm{h}} 31^{\mathrm{m}} 36^{\mathrm{s}}, \delta 21^{\circ} 58^{\prime} 40^{\prime \prime}$ and $\alpha 05^{\mathrm{h}} 31^{\mathrm{m}} 34^{\mathrm{s}}$, $\delta 21^{\circ} 59^{\prime} 20^{\prime \prime}$, both of which lie in the great 'eastern fan' on Woltjer's map.

Detailed study of the p.a. on the two maps is rendered uncertain because of the different resolutions, but it is clear that rotation measures in excess of $300 \mathrm{rad} / \mathrm{m}^{2}$ are to be found in such areas. As a result of the rapid variation, the smoothing by the 7 " beam cuts down the apparent degree of polarization considerably. Thus the high rotation measures mentioned by Burn refer only to the edge regions, and those close to prominent filaments. In contrast, the central area appears to have rather uniform rotation measure, close to the galactic value of $-25 \mathrm{rad} / \mathrm{m}^{2}$.

The degree of polarization at $\lambda 11 \mathrm{~cm}$ is about $10-11 \%$ near the centre, which is less than the optical polarization for the same area (25-30\%). Hence probably the Faraday mechanism is producing some depolarization on a small scale (less than the 7 " beam). It would seem that this depolarization is caused by the 'fibres' or fine filaments at the limit of seeing. If a magnetic field of the order of $300 \mu \mathrm{G}$ is assumed throughout the nebula, then these estimates of Faraday rotation suggest that the electron density is $\leqslant 3 \mathrm{~cm}^{-3}$ in the body of the nebula as a whole, but rises to perhaps 10 or 100 in the fibres and filaments. We may compare these with the value of 1500 $\mathrm{cm}^{-3}$ given by Woltjer for 'a few strong filaments'.

\section{References}

Allen, R. J.: 1967, Ph.D. Thesis, Massachusetts Institute of Technology.

Burn, B. J.: 1966, Monthly Notices Roy. Astron. Soc. 133, 67.

Downs, G. S. and Thompson, A. R.: 1968, Astrophys. J. 152, L65.

Gardner, F. F. and Davies, R. D.: 1966, Australian J. Phys. 19, 29.

Gardner, F. F. and Whiteoak, J. B.: 1966, Ann. Rev. Astron. Astrophys. 4, 245.

Mayer, C. H. and Hollinger, J. P.: 1967, Astrophys. J. 151, 53.

Wright, M.: 1970, Monthly Notices Roy. Astron. Soc. 150, 271.

\section{Discussion}

$N$. Visvanathan: The optical polarization is uniform within $15 \mathrm{sec}$ of arc of the pulsar. Is the same true of the radio polarization?

$R$. G. Conway: The optical polarization is not affected by Faraday rotation which causes small scale structure in the radio polarization. 